\title{
Survey on Evidence of Retrievability Schemes of Cloud Storage Services for Resource-Strained Devices
}

\author{
Ujjwala Bandawane $^{1}$, Sandeep Gore ${ }^{2}$ \\ ${ }^{1}$ G. H. Raisoni College of Engineering, Pune, Maharashtra, India \\ ${ }^{2}$ Assistant Professor, G. H. Raisoni College of Engineering, Pune, Maharashtra, India
}

\begin{abstract}
Cloud computing moves the application programming and databases to the concentrated vast server farms, where the administration of the information and administrations may not be completely reliable. Cloud storage service is the cloud administrations which can give an enormous storage space to solve the bottleneck of the storage space of local end clients. On the other hand, cloud storage administration may have information security because the clients' information is not stored in their own storage. To diminish the computational expense at client side amid the trustworthiness confirmation of their information, the idea of public verifiability has been proposed. Then again, the challenge is that the computational weight is excessively enormous for the clients with resourceconstrained devices to compute the public verification tags of file blocks. In this paper, author will concentrate on data integrity in the cloud storage administration. Public auditability is a model of outsourcing information uprightness check, which can accomplish productivity and security and survey on different resource-constrained devices for retrievability in cloud computing and compare them. In this way, review the past researches of data integrity based on public auditability which incorporates gathering the essential prerequisites and assessment measurements, giving the agent ways to deal with dissect security and effectiveness.
\end{abstract}

Keywords: Cloud storage, integrity, auditing, and proof of retrievability.

\section{Introduction}

Cloud computing has been imagined as the next generation design of the IT endeavor because of its long rundown of uncommon points of interest: on-demand selfadministration, ubiquitous system access, locationindependent asset pooling, quick asset flexibility, and use based valuing. Specifically, the everless expensive and all the more intense processors, together with the "software as a service" (SaaS) computing design, are changing server farms into pools of computing service on an immense scale.

Although having appealing advantages as a promising administration stage for the Internet, this new information storage paradigm in "cloud" brings numerous testing issues which have significant impact on the convenience, dependability, adaptability, security, and execution of the general framework. One of the greatest concerns with remote data storage is that of information respectability confirmation at untrusted servers. Case in point, the storage service provider may choose to cover up such information loss episodes as the Byzantine disappointment from the clients to maintain a reputation. Likewise genuine is that for saving money and storage space the service provider may intentionally dispose of once in a while got to information records which have a place with a normal customer. Considering the expansive size of the outsourced electronic information and the customer's compelled asset capacity, the center of the issue can be summed up as in what manner can the customer locate a proficient approach to perform periodical trustworthiness check without the neighborhood duplicate of information documents.

Keeping in mind the end goal to beat this issue, numerous plans have been proposed under diverse framework and security models. In every one of these works, awesome endeavors have been made to plan arrangements that meet different necessities: high plan proficiency, stateless verification, unbounded utilization of inquiries and retrievability of information, and so on. As indicated by the part of the verifier in the model, every one of the plans accessible falls into two classes: private verifiability and public verifiability. Albeit accomplishing higher productivity, plans with private evidence force computational weight on customers. Then again, public verifiability alleviates clients from performing a lot of calculation for guaranteeing the honesty of information storage. To be particular, customers have the capacity to delegate a third party to perform the check without dedication of their calculation assets. In the cloud, the customers may crash unexpectedly then again can't bear the cost of the overload of incessant respectability checks. Along these lines, it appears to be more normal and down to earth to prepare the check convention with open obviousness, which is normal to assume a more vital part in accomplishing better proficiency for cloud computing.

Additionally, there is another significant concern among past plans that is the support of dynamic data operation for cloud data storage applications. In cloud registering, the remotely put away electronic information may not just be gotten to additionally be redesigned by the customers, e.g., through block modification, deletion, insertion and so on. Tragically, the-state-of-the-art in the setting of remote information capacity principally concentrate on static information records and this element information redesigns has gotten constrained consideration in the information ownership applications so far. Despite the fact that such issue likewise has been tended to in various papers, it is very much trusted that supporting element information operation can be of

\section{Volume 4 Issue 12, December 2015}




\section{International Journal of Science and Research (IJSR) \\ ISSN (Online): 2319-7064}

Index Copernicus Value (2013): 6.14 | Impact Factor (2014): 5.611

imperative significance to the functional utilization of capacity outsourcing administrations. In perspective of the key part of public verifiability and dynamic information operation support for cloud information storage, This paper introduce a structure also, a productive development for consistent joining of these two parts in their convention plan. In expansion, the greater part of existing works embraces weaker security models which don't consider the reset attack. In particular, the cloud storage server (CSS) can trigger reset attacks in the transfer stage to damage the soundness of the plan.

\section{Literature Survey}

\subsection{Public Auditing of Dynamic Data}

In article [7] author proposed a novel dependable and secure information storage scheme with dynamic integrity assurance. Based on the principle of secret sharing and eradication coding, they first propose a hybrid share generation and distribution plan to accomplish dependable and fault tolerant providing so as to beg data storage excess for unique information parts. To encourage dynamically ensure the integrity of the distributed data shares; they then propose an effective data integrity verification plan exploiting the methods of algebraic signature and spotchecking. The proposed plan empowers singular sensors to check in one convention execution the correctness of all the pertaining data shares simultaneously in the absence of the original data. Broad security examination demonstrates that the proposed plan has solid resistance against different information contamination attacks. The productivity of the plan is shown by analyses on sensor stages Tmote Sky and iMote2.

In [8], Wang et al. thought of dynamic information storage in distributed situation, and also the projected challengeresponse protocol will each confirm the information correctness and find doable errors. Just like [10], they solely thought of partial support for dynamic information operation. In [5], they conjointly thought of the way to save space for storing by introducing deduplication in cloud storage. Recently, Zhu et al. [6] introduced the demonstrable information possession drawback in exceedingly cooperative cloud service suppliers and designed a replacement remote integrity checking system.

[1] Multi-proxy signature plans are exceptionally helpful devices when an original signer needs to delegate his signing ability to gathering of proxy signers, and have been recommended in various applications. The proxy revocation issue is a fundamental issue of the proxy signature schemes, on the other hand, it is rarely considered in the multi-proxy signature schemes. In this paper, author give a formal definition and security model of the multi-proxy signature schemes with proxy revocation, and propose a multi-proxy signature scheme with proxy revocation. Their plan can perform the prompt revocation by utilizing a security mediator (SEM), who inspects whether every proxy signer signs as per a warrant or its identity exists in a revocation list, and afterward chooses in the event that it issues a proxy token for every proxy signer. The proposed plan is demonstrated existentially unforgivable against picked message/warrant attacks based on the computational Diffie-
Hellman intractability assumption in the standard model. Besides, the measure of a multi-proxy signature is steady and independent of the number of the proxy signers.

[2] In this work, author contemplates the issue of ensuring the integrity of information storage in Cloud Computing. To reduce the computational expense at client side amid the trustworthiness confirmation of their information, the thought of public verifiability has been proposed. On the other hand, the test is that the computational weight is excessively enormous for the clients, making it impossible to compute the public authentication tags of file blocks. To handle the challenge, author propose another cloud storage structural planning with two autonomous cloud servers, that is, the cloud storage server and the cloud review server, where the recent is thought to be semi-honest. Specifically, they consider the errand of permitting the cloud review server, in the interest of the cloud clients, to pre-process the information before transferring to the cloud storage server and later checking the information respectability. The presentation of cloud review server dispenses with the contribution of client in the evaluating and in the preprocessing stages.

Liu et al. [15] think that past studies are not efficient in dynamic data update due to fixed-size block update. Along these lines, they propose a plan which can support variablesize blocks in dynamic data update. DR-DPDP is a plan that gives straightforward conveyance and replication of client information over different servers. There are three substances in the model. The customer, who stores information on the CSP, challenges the CSP to check the trustworthiness of information, and upgrades the store data [16].

\subsection{Public Auditing of Privacy Preserving Data}

[3] With cloud storage administrations, it is typical for information to be not only stored in the cloud, as well as shared over different clients. Then again, public auditing for such shared information - while protecting identity security - stays to be an open challenge. In this paper, author proposes the first privacy-preserving mechanism that permits public auditing on shared information stored in the cloud. Specifically, they endeavour ring signatures to register the confirmation data expected to review the trustworthiness of shared information. With their system, the identity of the signer on every piece in shared information is kept private from an third party auditor (TPA), who is still ready to publicly verify the integrity of shared information without recovering the whole document. Their exploratory results exhibit the efficiency and effectiveness of their proposed mechanism when auditing shared data.

\section{Volume 4 Issue 12, December 2015}




\section{International Journal of Science and Research (IJSR) ISSN (Online): 2319-7064}

Index Copernicus Value (2013): 6.14 | Impact Factor (2014): 5.611

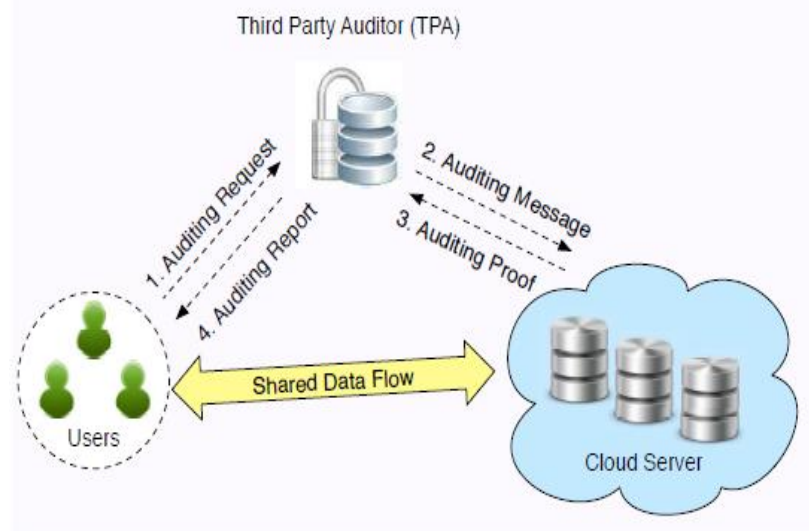

Figure 1: System model includes the cloud server, the third party auditor and users [3]

In this paper [4], the prevalence of this novel system in designing economical result confirmation algorithm for secure outsourcing is all around illustrated. Author diagnostically demonstrates that the proposed protocol at the same time satisfies the objectives of correctness, security, robust cheating resistance, and high-proficiency.

In this paper [11], author proposes a privacy-preserving public auditing framework for data storage security in Cloud Computing. Author use the homomorphic linear authenticator furthermore, random masking to ensure that the TPA would not realize any learning about the data content stored on the cloud server amid the efficient auditing procedure, which not just dispenses with the weight of cloud client from the dull and potentially extravagant inspecting errand, additionally mitigates the clients' fear of their outsourced data leakage.

Shacham and Waters [13] proposed an enhanced POR plan which employments BLS signature [14] to supplant the RSA-based signature to reduce the verification size. They utilize public verifiable homomorphic linear authenticators that are manufactured from BLS signature and secure random oracle model. They demonstrate that it is secure in a polynomial algorithm to reveal message.

The solution, as Shah et al. argue [20], is storage auditing: cryptographic systems that would allow users of outsourced storage services (or their agents) to verify that their data is still available and ready for retrieval if needed. Such a capability can be important to storage providers as well. Users may be reluctant to entrust their data to an unknown startup; an auditing mechanism can reassure them that their data is indeed still available.

\subsection{Public Auditing of Resource-constrained Devices}

Li et al. [12] proposed a public auditability plan in resourceconstrained devices. Li et al's. cloud information storage construction modeling. Resource-constrained device is a basic and lightweight composition. Subsequently, these devices have low computation and storage capacity. On the other hand, these devices can accomplish high mobility which permits clients to convey and effectively to utilization. Since the customer may require over and again modified information in cloud storage benefit, this operation needs to compute in each update.

Article [9] provides an associate economical verification theme for guaranteeing remote information integrity in cloud storage. The projected theme is established secure against reset attacks within the reinforced security model whereas supporting economical public verifiability and dynamic information operations at the same time projected a dynamic version of the previous PDP theme. However, the system imposes a priori certain on the quantity of queries and don't support absolutely dynamic information operations.

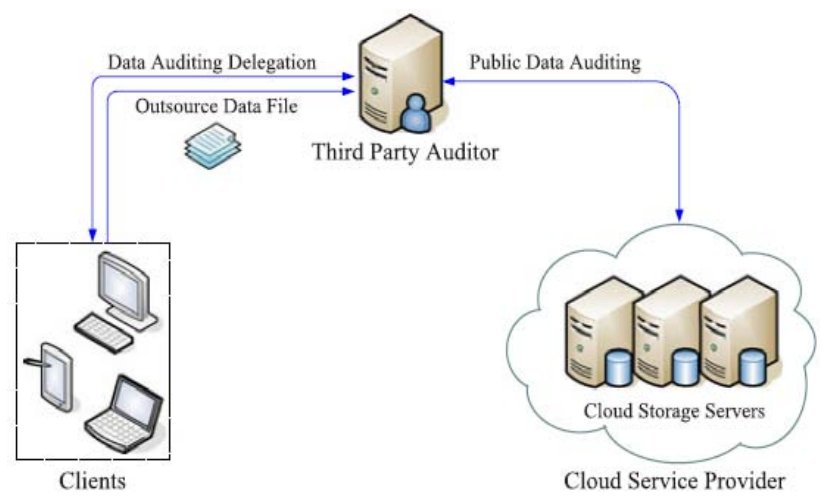

Figure 2: Cloud data storage architecture

[5] In this paper, author demonstrates, to some degree shockingly, that the two aspects can really exist together inside of the same system. This is conceivable on a very basic level on account of the accompanying knowledge: the public verifiability offered by PDP/POR schemes can be actually misused to accomplish POW. This "one stone, two birds" wonder not just motivated us to propose the novel idea of Proof of Storage with Deduplication (POSD), additionally guided us to outline a solid plan that is provably secure in the Random Oracle model taking based on the Computational DiffieHellman (CDH) suspicion.

[6] Provable data possession (PDP) is a method for guaranteeing the integrity of data in storage outsourcing. In this paper, author address the development of a proficient PDP plan for appropriated cloud storage to support the scalability of service and data migration, in which they consider the presence of different cloud service providers to helpfully store and keep up the clients' data. They exhibit a cooperative PDP (CPDP) plan taking into account homomorphic certain reaction and hash index hierarchy. They demonstrate the security of their plan based on multiprover zero-learning evidence framework, which can fulfil completeness, knowledge soundness, and zeroknowledge properties. Likewise, they explain performance optimization mechanisms for their plan, and specifically introduce an effective technique for selecting ideal parameter qualities to minimize the processing expenses of customers and storage service providers. Their analyses demonstrate that their answer presents lower computation and communication overheads in examination with non cooperative methodologies.

Ateniese et al. [17] proposed the provable data possession (PDP) model which can give public auditability and guarantee possession of files on untrusted capacity. They

\section{Volume 4 Issue 12, December 2015}




\section{International Journal of Science and Research (IJSR) \\ ISSN (Online): 2319-7064}

Index Copernicus Value (2013): 6.14 | Impact Factor (2014): 5.611

utilize RSA-based homomorphic verifiable labels to review outsourced information. Their plan first gives blockless verification and open verifiability at the same time. Be that as it may, Ateniese et al's. plan can't support dynamic data verification on the grounds that their plan just considers static information circumstance which implies the customer stores outsourced information and won't adjust it. In this way, Ateniese et al. [18] proposed an adaptable PDP plan to enhance dynamic information verification in 2008. By and by, their plan can't support completely dynamic information which can't support block insertions because their scheme only allows simple block operation which implies partially dynamic data like block modification and block deletion.

A -spt-checking" mechanism is being used in the challenge-response protocol to detect adversarial behavior. In each challenge, a subset of file blocks is sampled, and the results of a computation over these blocks is returned to the client. The returned results are checked using some additional information embedded into the file at encoding time. [19]

\section{Comparative Analysis}

Since clients' information is stored in the cloud storage service, it brings clients' information security issues. In the public auditability model, clients can appoint the third party auditor to confirm their information is effective. As per the literature this paper sort out the essential necessities in public auditability, which can be arranged to the case for the application.

According to previous studies, where they provide the basic requirements of security and performance. This paper classifies and describes these requirements.

1)Blockless Verification: - The reviewer can confirm data blocks, and need not to recover all evaluated data blocks in the distributed storage service.

2)Stateless Verification: - The auditor needs not to maintain and update data situation because data situation is maintained by the client and cloud storage service together.

3)Batch Auditing:- The auditor can verify the data of different clients at the same time because the auditor can be delegated by a lot of clients.

4)Dynamic Data:- The data owner can insert, modify and delete data blocks in the cloud storage.

\begin{tabular}{|c|c|c|c|c|c|c|}
\hline Methods & {$[\mathbf{3}]$} & {$[\mathbf{7 ]}$} & {$[\mathbf{2 ]}$} & {$[\mathbf{9 ]}$} & {$[\mathbf{1 1}]$} & {$[\mathbf{1 2}]$} \\
\hline Blockless verification & Yes & Yes & Yes & Yes & Yes & Yes \\
\hline Stateless verification & No & Yes & No & Yes & Yes & Yes \\
\hline Batch auditing & Yes & Yes & Yes & Yes & Yes & Yes \\
\hline Dynamic data & Partial & Partial & Partial & Yes & Partial & Yes \\
\hline Privacy presenting & Yes & No & No & Yes & Yes & No \\
\hline
\end{tabular}

\section{Conclusion}

This paper survey on different resource-constrained devices for retrievability in cloud computing and conclude that no existing plan can simultaneously provide provable security in the improved security model and appreciate attractive proficiency, that is, no plan can oppose reset attacks while supporting productive public verifiability and dynamic data operations at the same time. So their needs have proposed a model new confirmation of retrievability for cloud storage, in which a reliable review server is introduced with preprocess and transfer the information for the benefit of the clients. Furthermore the computation overhead for tag generation on the client side is diminished fundamentally. The cloud review server likewise performs the data integrity verification or updating the outsourced information upon the clients' request.

\section{References}

[1] Z. Liu, Y. Hu, X. Zhang, and H. Ma, Provably secure multi-proxy signature scheme with revocation in the standard model," Comput. Commun. vol. 34, no. 3, pp. 494-501, 2011.

[2] J. Li, X. Tan, X. Chen, and D. S. Wong, -Anefficient proof of retrievability with public auditing in cloud computing," in Proc. 5th Int. Conf. Intell. Netw. Collaborative Syst., 2013, pp. 93-98.
[3] H. Li, B. Wang, and B. Li, - Orta: Privacy-preserving public auditing for shared data in the cloud," IEEE Trans. Cloud Comput., vol. 2, no. 1, pp. 43-56, Jan.Mar. 2014.

[4] X. Lei, X. Liao, T. Huang, H. Li, and C. Hu, - Outsourcing large matrix inversion computation to a public cloud,", IEEE Trans. Cloud Comput., vol. 1, no. 1, p. 1, Jan.-Jun. 2013.

[5] Q. Zheng, and S. Xu, - Secre and efficient proof of storage with deduplication," in Proc. ACM Conf. Data Appl. Security Privacy, 2012, pp. 1-12.

[6] Y. Zhu, H. Hu, G.-J. Ahn, and M. Yu, -Coperative provable data possession for integrity verification in multicloud storage," IEEE Trans. Parallel Distrib. Syst., vol. 23, no. 12, pp. 2231-2244, Dec. 2012.

[7] Q. Wang, K. Ren, S. Yu, and W. Lou, Đependable and secure sensor data storage with dynamic integrity assurance," ACM Trans. Sens. Netw., vol. 8, no. 1, pp. 9:1-9:24, Aug. 2011.

[8] C. Wang, Q. Wang, and K. Ren, Ensuring data storage security in cloud computing," in Proc. 17th Int. Workshop Quality Serv., 2009,pp. 1-9.

[9] Jin Li, Xiao Tan, Xiaofeng Chen, Duncan S. Wong, and Fatos Xhafa" OPoR: Enabling Proof of Retrievability in Cloud Computing with Resource-Constrained Devices," IEEE Transactions on cloud computing, vol. 3, no. 2, April/June 2015.

[10]L. V. M. Giuseppe Ateniese, R. D. Pietro, and G. Tsudik, Scalable and efficient provable data 


\section{International Journal of Science and Research (IJSR) \\ ISSN (Online): 2319-7064}

Index Copernicus Value (2013): 6.14 | Impact Factor (2014): 5.611

possession," in Proc. Int. Conf. Security Privacy Commun. Netw., 2008, pp. 46-66.

[11]C. Wang, S. S. M. Chow, Q. Wang, K. Ren, and W. Lou, Privacy-preserving public auditing for secure cloud storage," IEEE Transactions on Computers, vol. 62, no. 2, pp. 362-375, 2013.

[12] J. Li, X. Tan, X. Chen, D. Wong, and F. Xhafa, “'OPoR: Enabling proof of retrievability in cloud computing with resource-constrained devices," accepted and to be publish in IEEE Transactions on Cloud Computing, Oct. 2014.

[13]H. Shacham and B. Waters, -Cmpact proofs of retrievability," in Proceedings of the 14th International Conference on the Theory and Application of Cryptology and Information Security (ASIACRYPT'08), pp 90-107, Melbourne, Australia, 2008.

[14] D. Boneh, B. Lynn, and H. Shacham, - Bort signatures from the weil pairing," in Proceedings of the 7th International Conference on the Theory and Application of Cryptology and Information Security: Advances in Cryptology (ASIACRYPT'01), pp. 514-532, Gold Coast, Australia, 2001.

[15]C. Liu, J. Chen, L. T. Yang, X. Zhang, C. Yang, R. Ranjan, and K. Ramamohanarao, Authorized public auditing of dynamic big data storage on cloud with efficient verifiable fine-grained updates," IEEE Transactions on Parallel and Distributed Systems, vol. 25, no. 9, pp. 2234-2244, 2014.

[16] Mohammad Etemad and Alptekin Koc University, Istanbul, Turkey." Transparent, Distributed, and Replicated Dynamic Provable Data Possession”.

[17] G. Ateniese, R. Burns, R. Curtmola, J. Herring, L. Kissner, Z. Peterson, and D. Song, Provable data possession at untrusted stores," in Proceedings of the 14th ACM Conference on Computer and Communications Security, pp. 598-609, Virginia, USA, 2007.

[18] G. Ateniese, R. D. Pietro, L. V. Mancini, and G. Tsudik, Scalable and efficient provable data possession," in Proceedings of the 4th International Conference on Security and Privacy in Communication Netowrks, pp. 9:1-9:10, Istanbul, Turkey, 2008.

[19] Kevin D. Bowers, Ari Juels, Alina Oprea, Proofs of Retrievability: Theory and Implementation, CCSW'09, Journal of Systems and Software, v.85 n.5, p.10831095, May, 2012.

[20] M. Shah, M. Baker, J. Mogul, and R. Swaminathan. Auditing to keep online storage services honest. In G. Hunt, editor, Proceedings of HotOS 2007. USENIX, May 2007 